\title{
LAS REVISTAS ESPECIALIZADAS: UN FONDO DOCUMENTAL PARA EL ESTUDIO DE LA HISTORIA DE LA DANZA EN ESPAÑA
}

\author{
Ana Isabel Elvira Esteban \\ Asociación Española Danza más Investigación ( $D+l)$, \\ Investigadora independiente.
}

\section{Resumen}

Dado el poco interés que la danza ha suscitado en España, a la hora de reconstruir su historia es insuficiente contar con la prensa cotidiana como única fuente secundaria, pues sus referencias suelen ser escasas, incompletas y en ciertas ocasiones erróneas. Para poder profundizar su estudio y contextualizarlo de forma adecuada, proponemos ayudarse de otro tipo de publicación no muy abundante, las revistas especializadas, con las que sí es posible trazar un itinerario temporal de nuestra danza desde la década de los 70 en el siglo XX en adelante. El presente artículo ofrece un recorrido por el panorama editorial de estas publicaciones, al tiempo que incide en la necesidad de rescatarlas y valorarlas como un importante fondo de documentación a tener en cuenta para la investigación histórica de esta disciplina artística en nuestro país.

\section{Palabras-clave: REVISTAS ESPECIALIZADAS EN DANZA; DOCUMENTACIÓN SOBRE DANZA; HISTORIA DE LA DANZA EN ESPAÑA}

\section{Abstract}

Given the lack of interest that in dance has raised in Spain, using the daily press as a secondary source to reconstruct its history it is insufficient, since references are often scarce, incomplete and in some cases erroneous. To deepen their study and properly contextualize propose help of specialized magazines, a type of not extensive publication, but with which it is possible to draw a temporary schedule of our dance from the 70 s in the twentieth century onwards. This article offers an overview of the publishing scene of these publications, while stressing the need to rescue them and value them as an important background documentation to be considered for historical research of this artistic discipline in our country.

\section{Keywords: SPECIALIZED MAGAZINES ON DANCE; DANCE DOCUMENTATION; HISTORY OF DANCE IN SPAIN}

\footnotetext{
Elvira Esteban, Ana Isabel. 2015. "Las revistas especializadas: un fondo documental para el estudio de la historia de la danza en España”. AusArt 3 (1): 244-256. D0I: 10.1387/ausart.14478
}

\section{AUSART}




\section{INTRODUCCIÓN}

La ausencia de publicaciones sobre la danza en España se debe, no sólo a la intrínseca dificultad de esta disciplina artística para ser conservada, sino también y a diferencia de otros países de su entorno, al escaso reconocimiento que la sociedad española ha mostrado hacia ella.

La escasez de estudios históricos sobre danza en nuestro país, es tan manifiesta, que ni siquiera a día de hoy es posible encontrar publicaciones que permitan tener una perspectiva histórica completa de su evolución. En las últimas décadas, gracias al cambio de enfoque educativo implantado a partir de la LOGSE (1992) y al empuje de ciertos sectores profesionales, se observa un giro positivo en este sentido y la danza comienza a ser considerada una materia interesante para ser analizada desde un punto de vista científico; razón por la cual, conviene tener en cuenta toda la información que permita recuperar esa inexistente, pero imprescindible visión global.

\section{OBJETIVOS}

El objetivo principal del presente artículo es ofrecer un recorrido por el panorama editorial de las publicaciones españolas especializadas en danza, y al tiempo incidir en la necesidad de rescatar y valorar este tipo de fuente secundaria como un importante fondo de documentación que ha de tenerse en cuenta a la hora de investigar sobre la historia de esta disciplina artística en nuestro país.

\section{METODOLOGÍA}

Metodológicamente este estudio se basa en la búsqueda y recopilación de información a partir de las mismas fuentes secundarias que se analizan, las revistas españolas especializadas en danza, a las que se suma otra documentación que ha podido recogerse a través de otras publicaciones periódicas (periódicos y revistas especializadas en otras disciplinas) o textos especiali- 
zados, para proceder después a su análisis, desde un enfoque metodológico cualitativo, y a la exposición razonada de la documentación que ha sido examinada ${ }^{1}$. Hemos elaborado una tabla compilatoria final, que facilite al lector una visión general del conjunto de las publicaciones (ver anexo).

\section{RESULTADOS Y DISCUSIÓN}

El 11 de enero de 1987, el crítico de danza Roger Salas comunicaba desde las páginas del diario El País la suspensión de Dansa79, "la única publicación española dedicada enteramente a la danza y el ballet", tras siete años ininterrumpidos. Sin embargo, no puntualizaba que sus directoras habían seguido los pasos de la también desaparecida La Danza, que podría ser considerada la primera iniciativa española en ese sentido.

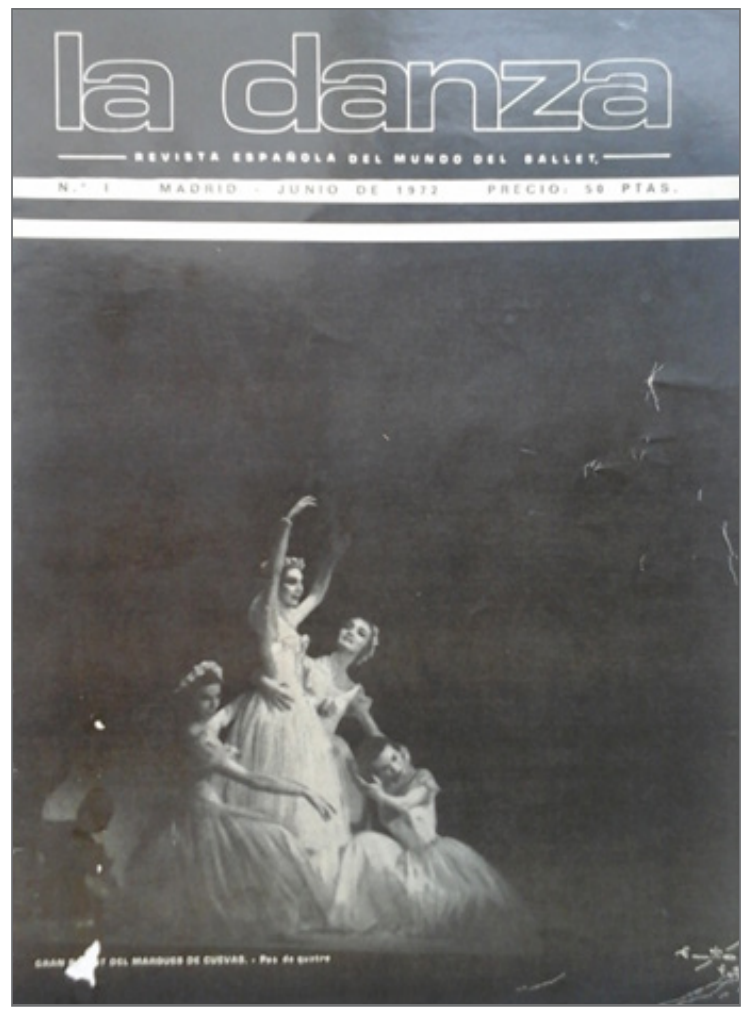

Figura 1. Portada de La Danza (n¹,1972) 
Subtitulada como Revista española del mundo del ballet e única en el espacio nacional en aquel tiempo, La Danza había sido promovida e impulsada por el empresario Marcos Menkes con la ayuda de un grupo de colaboradores y pretendía cubrir un "Ilamativo" hueco en el panorama de la danza. La editorial de su número inicial marcaba como objetivos principales la divulgación y la orientación desde una actitud crítica pero sin perder el espíritu constructivo. Su sede se encontraba en Madrid y tuvo una corta trayectoria: desde su primer número fechado en junio de 1972 hasta el último de noviembre de 1974 (ver tabla1).

El relevo lo tomaron Beatriu Daniel, Montse García Otzet y Carme del Val, directoras iniciales de Danza 79, aunque en esta ocasión su ubicación fuera otra. Desde Barcelona, y a instancias del maestro Joan Tena (García Otzet 1993, 118-22), se embarcaron en un primer boletín divulgativo fechado en enero de 1979, que tomó forma y creció hasta convertirse en una revista especializada en febrero de $1981^{2}$. Aunque las fuerzas se mermaron por la marcha de Daniel, sus responsables lograron mantener la actividad de esta revista hasta el número 51, y lo hicieron gracias al impulso sin precedentes que tuvo la creación coreográfica española en esa década.

Cataluña se destacó en esos primeros años 80 como uno de los motores de la danza española. Eso explicaría que tan sólo un mes después de la llegada de Dansa 79, apareciera allí una nueva publicación dancística, aunque esta vez ligada a la revista de música Monsalvat. Lo hizo en forma de separata acompañando al número 58. Había sido anunciada por su director, José Manuel Infiesta en otro número anterior, el 51, que ya contenía un apartado exclusivo de danza de 17 páginas. Pastora Martos, bailarina, maestra y coreógrafa, lideró esta idea en su calidad de jefa de redacción. No era su primera experiencia en estas lides, pues ya en enero de 1978 había puesto en marcha un boletín de danza (Aviñoa 1987, 240), del que se editaron 6 números. La trayectoria de Montasalvat Danza fue larga, hasta 1992, según apunta Gala Vivancos (2009) en la web http://babourc.eu/Resources/dectatoma/todoarte pastora martos. htm, aunque en su evolución dejó de editarse por separado y se integró como sección de la revista.

Otras revistas especializadas de música mostraron su interés por incorporar información sobre danza a sus lectores, probablemente debido a la cercanía entre ambas disciplinas escénicas, e incluso también a causa del palpable aumento de actividades dancísticas durante las siguientes décadas. Así, aunque lo hicieron con posterioridad a Montsalvat, si revisa con detalle su conte- 


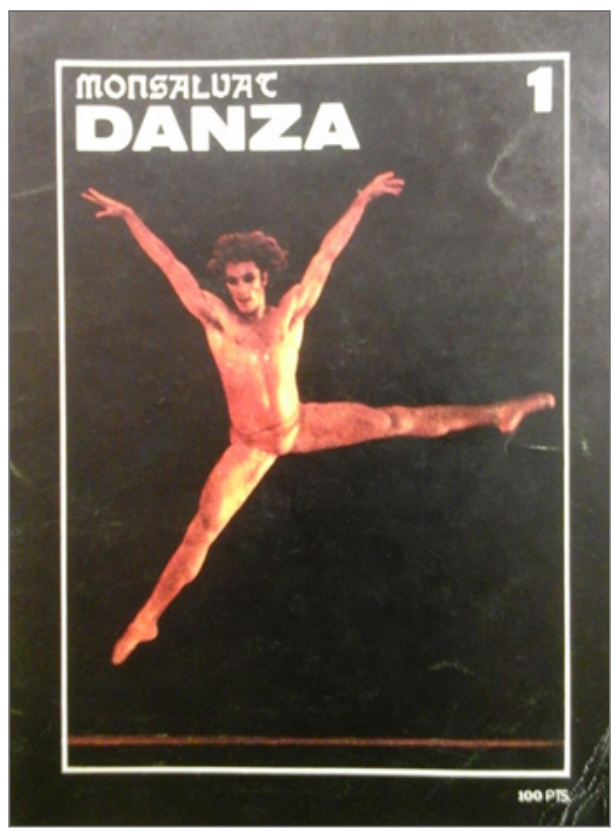

Figura 2. Portada del Montsalvat Danza ( $\left.n^{\circ} 1,1979\right)$

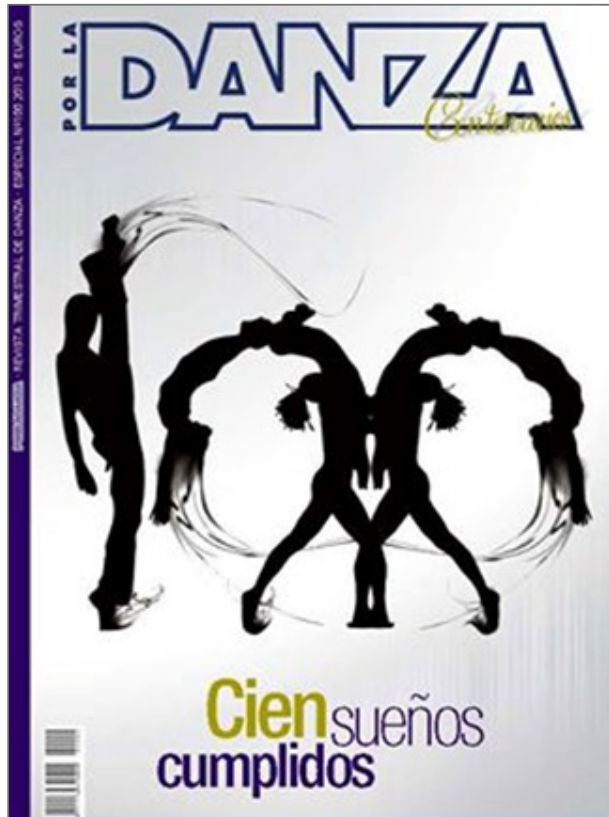

Victor Ullate - Jean Christophe Maillot - It Dansa

Figura 3. Portada de Por la Danza (n 100 , otoño 2013) nido, es posible encontrar mucha información en Ritmo, Scherzo y Melómano.

La andadura de Por la Danza, publicación ligada en su origen a la Asociación de Profesionales de la Danza en la Comunidad de Madrid y a la Asociación Cultural Por la Danza, también se inició en 1987 a partir de un boletín informativo para socios y no fue hasta diciembre de 1989 cuando apareció la revista con un primer número 0 . Comparativamente hablando, es la revista especializada en danza de más largo recorrido y que ha logrado una mayor continuidad en el tiempo. A lo largo de su camino ha sufrido importantes transformaciones de tamaño y apariencia, grafismo, contenidos, así como de los colaboradores que han contribuido a completar sus contenidos. Ha superado ya el centenar de ediciones y mantiene su actividad.

Resulta curioso comprobar cómo en septiembre de 1992, el crítico de danza Julio Bravo, anunciaba en ABC la salida del segundo número de la revista Ballet 2000, como "la primera publicación española dedicada exclusivamente a la danza desde la desaparición de Dansa 79". Por la Danza ya existía, pero dada su relación directa con una asociación profesional, deducimos que se la consideraba poco independiente desde un punto de vista periodístico y no se la tuvo en cuenta.

Dicho esto, es evidente que la propuesta de Ballet 2000, cuyo primer número salió en junio de 1992, supe- 
raba con creces a cualquier otra, pues se trataba de un proyecto trasnacional tutelado por Alfio Agostini, director y editor al mismo tiempo de la italiana Balletto Oggi y la francesa Ballet 2000. El ambicioso planteamiento inicial, tal y como se recoge en la editorial del primer número de la edición española, era mirar más allá de las fronteras y superar el provincianismo de la crítica de danza aprovechando la fuerza de una red informativa cooperativa. En las tres revistas se compartían ciertos contenidos comunes y se incorporaba la información de interés nacional a la edición de cada país, fundamentalmente en el apartado de noticias breves. Fue un proyecto interesante que se mantuvo hasta 1998, aunque sufrió ciertos reajustes en los números más inmediatos a su salida debido al aumento de costes.

\section{CAIRON}

Revista de Ciencias de la Danza

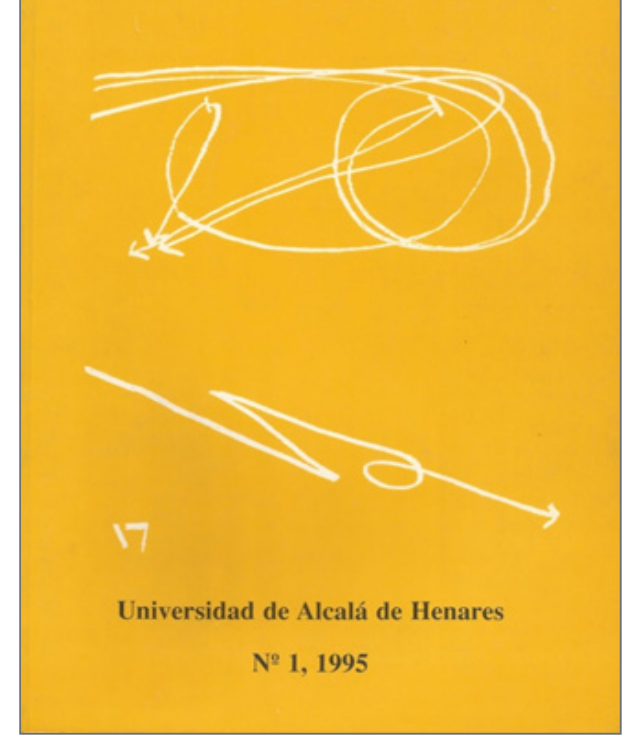

Figura 4. Portada de Cairón. Revista de Ciencias de la Danza $\left(n^{\circ} 1,1995\right)$

Durante la década de los años 90, hubo alguna apuesta más por sacar al mercado nuevas publicaciones especializadas, a las que hay que sumar los boletines informativos que comenzaron a editarse con efervescencia desde las asociaciones de profesionales de la danza ${ }^{3}$. Una de esas propuestas fue Dansart, una revista trilingüe en castellano, catalán e inglés. Sus editoras indicaban como objetivos de su proyecto el ofrecer documentación y dejar huella de nuestra historia, al mismo tiempo que informar, compartir vivencias y experiencias y fomentar la difusión de textos escritos en castellano y catalán. No logró permanecer en el tiempo, posiblemente por tratarse de una idea excesivamente costosa. Tampoco lo lograron otras pequeñas iniciativas, como la de la revista ;Bailar! (ver tabla 1), quizás por tener una trascendencia excesivamente local. Por el contrario, sí alcanzó un interesante desarrollo el proyecto del Aula de Danza de la Universidad de Alcalá de Henares, liderado por Estrella Casero, que logró poner en marcha la primera revista española de investigación sobre danza: Cairón. Revista de Ciencias de la Danza. Su objetivo fundamental, tal y como indicaba el rector de la UAH en la introducción de su número 0 , era "convertir a la danza en una disciplina universitaria". No podemos olvidar que en esos años, ya había en España una enorme inquietud por la investigación dentro de pequeños círculos del ámbito profesional dan- 
cístico y se habían defendido las primeras tesis doctorales que investigaban sobre esta materia (Elvira, "La investigación en danza. ¿Hacia dónde vamos?”, 2012).

La llegada del nuevo milenio significó una cierta explosión de revistas de danza, que se multiplicaron por toda España, muchas de ellas ligadas a asociaciones o entidades sin ánimo de lucro, e incluso algunas gratuitas. Así surgieron Zaradanza en Zaragoza en abril de 2002; Danza en Escena en Logroño, cuyo primer número está fechado entre julio y septiembre de 2003; Con D de Danza en Valencia, que apareció el mismo año en el mes de octubre; Tiempo de Danza en Murcia, que inició sus primeros pasos en febrero de 2005 e Inquedanzas, que lo hizo en julio de 2006. Otras nacieron a partir de iniciativas privadas, como Susy $Q$, cuyo primer número salió en noviembre de 2005 y es una de las pocas revistas que continúan vigentes a día de hoy, o Danza Ballet, una publicación de colección, casi de lujo, que apareció en agosto de 2011 y de la que sólo se editaron 5 números. Entre las que aún permanecen en el mercado quedan por señalar Danza Europa, un proyecto europeo que apostó por el mercado de lengua castellana y sacó una edición mensual española en 2009, y E-danza.net, una revista en formato digital que se ofrece desde un portal virtual dedicado a la danza ${ }^{4}$.

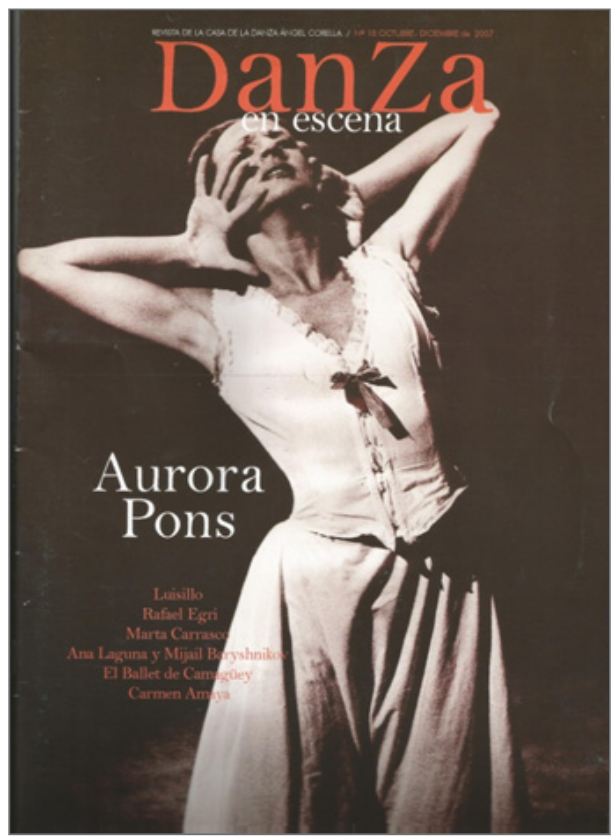

Figura 5. Portada de Danza en Escena (n¹8, 2007)
En la primera década del 2000 y siguiendo los pasos de Cairón, también aparecieron varias publicaciones con un perfil menos informativo y más reflexivo: Diálogos de Danza en Valencia, El Círculo de la Danza en Madrid, Danzaratte en Málaga y En Moviment en Barcelona. La única que permanece activa, aunque su salida sea bastante irregular, es Danzaratte, que se ha ofrecido en formato digital desde el primer número.

Como ya hemos mencionado y al igual que lo hizo Monsalvat en la década de los 70 , otras revistas especializadas en música dedicaron parte de sus páginas a la danza. Melómano incluso, comenzó un proyecto para crear un suplemento 
exclusivo de danza coordinado por la periodista y crítica de danza Cristina Marinero ${ }^{5}$. Del mismo modo otras revistas relacionadas con distintas artes escénicas también han hecho guiños a la danza y han mostrado su interés por ofrecer información sobre sus actividades; entre ellas El Público, ADE o El Pateo.

No queremos cerrar este artículo sin dejar de hacer mención a publicaciones como Revista de Folklore o La Tarasca, en las que es posible encontrar información y reflexiones interesantes relacionadas con la danza tradicional. $Y$ aunque desarrolle su actividad exclusivamente en el País Vasco, merece especial atención una de las decanas, Dantzariak, nacida en 1966 por iniciativa de Euskal Dantzarien Biltzarra (Asociación de Dantzaris) para preservar, mantener y conservar la danza tradicional vasca.

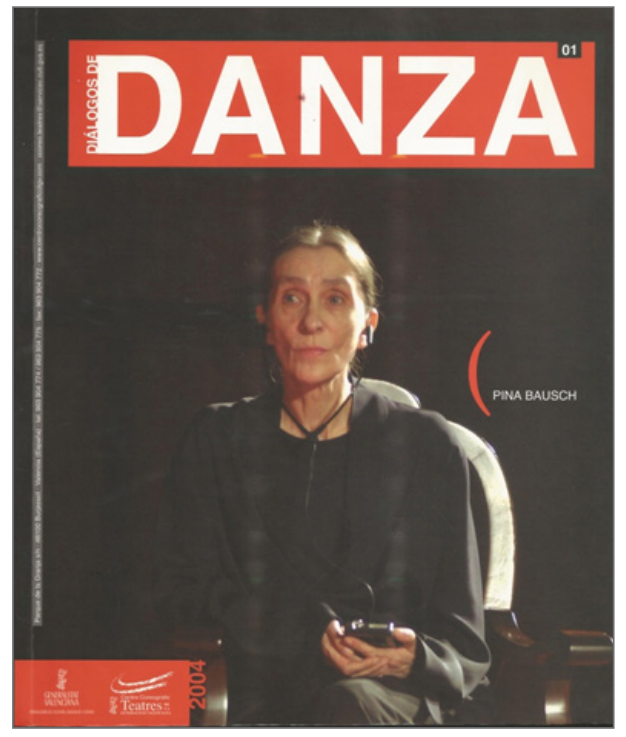

Figura 6. Portada de Diálogos de Danza (n¹, 2004)

\section{CONCLUSIONES}

Pese al cierto desánimo que puedan provocar las palabras de Roger Salas en su artículo de El País “¿Cómo se escribe la historia de la danza?” (2013), cuando manifiesta y hace evidente la casi completa desaparición de muchas de las revistas de danza que han ido surgiendo en los últimos treinta años, no hay duda de que el panorama de estas revistas especializadas en España, desde la década de los 70 hasta el día de hoy, ha alcanzado una continuidad en el tiempo. De acuerdo a nuestro objetivo inicial, en este artículo hemos intentado presentar un bosquejo ordenado de las más destacadas y conocidas, aunque sabemos que no están recogidas todas.

Sin olvidar que los espacios vacíos y las lagunas de información pueden ser muchos y evidentes, entendemos que es muy necesario reivindicar el uso de este tipo de publicaciones como fuente documental para el estudio y la investigación por dos razones fundamentales: 
1. Porque permiten disponer de una información relacionada con el sector profesional dancístico imposible de encontrar en otras, como periódicos o textos especializados, ya sea a través de anuncios y agendas, como de entrevistas, noticias breves, críticas, artículos, cartas al director, información bibliográfica y de vídeo, etc.

2. Porque pueden contribuir a la reconstrucción documentada de esa historia de la danza todavía pendiente en nuestro país, pese a que los primeros resultados no aporten más que un armazón hueco pendiente de ser cubierto. Pero ése ya sería un interesante punto de partida.

Esperamos que la exposición secuenciada y temporal de su aparición en el panorama editorial español haya facilitado una mejor comprensión de las fuentes documentales citadas y se entienda la división en dos grupos diferenciados en función de su objetivo: las que se dedican fundamentalmente a informar y divulgar, y las que se inclinan más hacia la reflexión y la investigación.

Es cierto que queda pendiente por hacer lo más importante, el análisis detallado de sus contenidos, sus colaboradores y su evolución individualizada, pero eso se abordará en un futuro próximo. De lo que no hay duda, es que este tipo de publicaciones comienzan a suscitar mucho interés, especialmente en los últimos años, y algunos ejemplos de ello son la inclusión de todos los números digitalizados de Dansa 79 en $\mathrm{ARCA}^{6}$ o la publicación de estudios como el realizado por Brozas en 2011, "La danza contemporánea en España (1989-2009)", cuya fuente documental básica fue la revista Por la Danza. 


\section{ANEXO}

\begin{tabular}{|c|c|c|c|c|c|}
\hline Nombre & $\begin{array}{l}\text { Mes-Trim.l } \\
\text { Año inicio }\end{array}$ & $\begin{array}{l}\text { Mes/Año } \\
\text { cierre }\end{array}$ & $\begin{array}{l}\text { Nros. } \\
\text { publi- } \\
\text { cados }\end{array}$ & $\begin{array}{l}\text { Editor y/o } \\
\text { entidad } \\
\text { editora }\end{array}$ & $\begin{array}{l}\text { Director-a y/o } \\
\text { Coordinador-a } \\
\text { inicial }\end{array}$ \\
\hline \multicolumn{6}{|c|}{ PUBLICACIONES INFORMATIVAS Y DE DIVULGACIÓN } \\
\hline $\begin{array}{l}\text { La Danza. } \\
\text { Revista } \\
\text { española del } \\
\text { mundo del } \\
\text { ballet. }\end{array}$ & $6 / 1972$ & $11 / 1974$ & 17 & & $\begin{array}{l}\text { Marcos } \\
\text { Menkes }\end{array}$ \\
\hline $\begin{array}{l}\text { Boletín de } \\
\text { Danza }\end{array}$ & $1 / 1978$ & 1993 & 6 & & Pastora Martos \\
\hline Dansa 79 & $1 / 1979$ & $10 / 1986$ & 51 & & $\begin{array}{l}\text { Beatriu Daniel, } \\
\text { Montse Ga. } \\
\text { Otzet y Carme } \\
\text { del Val }\end{array}$ \\
\hline $\begin{array}{l}\text { Monsalvat } \\
\text { Danza }\end{array}$ & $2 / 1979$ & 1992 & & $\begin{array}{l}\text { Ediciones } \\
\text { de nuevo } \\
\text { arte Thor }\end{array}$ & $\begin{array}{l}\text { José Manuel } \\
\text { Infiesta/ } \\
\text { Pastora Martos }\end{array}$ \\
\hline $\begin{array}{l}\text { Boletín de } \\
\text { danza }\end{array}$ & 1987 & 1988 & 7 & APDCM & Javier Bagá \\
\hline Por la Danza & $12 / 1989$ & vigente & $\begin{array}{c}106+ \\
\mathrm{n}^{\circ} 0\end{array}$ & $\begin{array}{l}\text { APDCM y/ } \\
\text { ACPD }\end{array}$ & Enrique Brown \\
\hline Ballet 2000 & $6 / 1992$ & 1998 & & $\begin{array}{l}\text { Alfio } \\
\text { Agostini }\end{array}$ & $\begin{array}{l}\text { Roger Salas } \\
\text { (coordinador } \\
\text { de la edición } \\
\text { española) }\end{array}$ \\
\hline ¡Bailar! & $2 / 1993$ & $5-6 / 1993$ & $4+n^{\circ} 0$ & $\begin{array}{l}\text { Escuela } \\
\text { de danza } \\
\text { Duque }\end{array}$ & $\begin{array}{l}\text { Cristina } \\
\text { Marinero/ } \\
\text { Alessandro } \\
\text { Pierozzi }\end{array}$ \\
\hline Dansart & otoño 1997 & $\begin{array}{l}\text { Primavera } \\
\text { / verano } \\
2000 \\
\end{array}$ & 3 & $\begin{array}{l}\text { Los libros } \\
\text { de la danza, } \\
\text { S.L. }\end{array}$ & $\begin{array}{l}\text { Nélida Monés y } \\
\text { Marta Carrasco }\end{array}$ \\
\hline Zaradanza & 4 / 2002 & & & $\begin{array}{l}\text { Asociación } \\
\text { cultural } \\
\text { Zaradanza }\end{array}$ & $\begin{array}{l}\text { José Miguel } \\
\text { González }\end{array}$ \\
\hline $\begin{array}{l}\text { Danza en } \\
\text { Escena }\end{array}$ & 7- 9 / 2003 & vigente & $\begin{array}{c}38+1 \\
n^{\circ} \\
\text { espec. }\end{array}$ & $\begin{array}{l}\text { Casa de } \\
\text { la Danza } \\
\text { (Logroño) }\end{array}$ & $\begin{array}{l}\text { Perfecto Uriel/ } \\
\text { Mila Ruiz }\end{array}$ \\
\hline
\end{tabular}




\begin{tabular}{|c|c|c|c|c|c|}
\hline $\begin{array}{l}\text { Con D de } \\
\text { Danza }\end{array}$ & $10 / 2003$ & $7 / 2011$ & 28 & $\begin{array}{l}\text { C.S.I. } \\
\text { Comuni- } \\
\text { cación } \\
\text { publicitaria }\end{array}$ & $\begin{array}{l}\text { Cristina Soler } \\
\text { Ibermón }\end{array}$ \\
\hline $\begin{array}{l}\text { Tiempo de } \\
\text { Danza }\end{array}$ & $2-3 / 2005$ & $\begin{array}{c}12-1 / 2010- \\
11\end{array}$ & $\begin{array}{l}23+ \\
n^{\circ} 0\end{array}$ & $\begin{array}{l}\text { Asociación } \\
\text { Amigos de } \\
\text { la Danza- } \\
\text { Universidad } \\
\text { de Murcia }\end{array}$ & $\begin{array}{l}\text { Margarita } \\
\text { Muñoz Z./ } \\
\text { Georgina } \\
\text { Cayuela }\end{array}$ \\
\hline Susy $Q$ & $\begin{array}{l}11-12 / \\
2005\end{array}$ & vigente & $\begin{array}{c}53+ \\
n^{\circ} 0+ \\
n^{o s} \\
\text { espec. }\end{array}$ & $\begin{array}{l}\text { Editorial } \\
\text { Isadora, } \\
\text { S.L. }\end{array}$ & $\begin{array}{l}\text { Omar Khan/ } \\
\text { Mercedes } \\
\text { López } \\
\text { Caballero }\end{array}$ \\
\hline Inquedanzas & $7-8 / 2006$ & $1-2 / 2008$ & $6+n^{\circ} 0$ & AGPD & $\begin{array}{l}\text { Begoña Acuña } \\
\text { (coordinadora) }\end{array}$ \\
\hline $\begin{array}{l}\text { Danza } \\
\text { Europa }\end{array}$ & 2009 & vigente & & $\begin{array}{l}\text { Emma } \\
\text { Maning }\end{array}$ & Elia Luyando \\
\hline $\begin{array}{l}\text { e-danza.net. } \\
\text { www.edanza. } \\
\text { net }\end{array}$ & $3-4 / 2010$ & vigente & 30 & G.I. Eter & $\begin{array}{l}\text { Emilio Tenorio/ } \\
\text { Virginia G. } \\
\text { Alarcos }\end{array}$ \\
\hline $\begin{array}{l}\text { Danza Ballet. } \\
\text { Revista de } \\
\text { colección }\end{array}$ & $8 / 2011$ & $10 / 2012$ & 5 & $\begin{array}{l}\text { Carolina } \\
\text { de Pedro } \\
\text { Pascual }\end{array}$ & $\begin{array}{l}\text { Ruben Wortel/ } \\
\text { Carolina de } \\
\text { Pedro Pascual }\end{array}$ \\
\hline \multicolumn{6}{|c|}{ PUBLICACIONES DE REFLEXIÓN YIO INVESTIGACIÓN } \\
\hline Cairón & 1995 & 2012 & 14 & $\begin{array}{l}\text { Aula de } \\
\text { Danza UAH }\end{array}$ & Delfín Colomé \\
\hline $\begin{array}{l}\text { Diálogos de } \\
\text { Danza }\end{array}$ & 2004 & $2005 ?$ & 2 & $\begin{array}{l}\text { Centro } \\
\text { Coreo- } \\
\text { gráfico TGV }\end{array}$ & $\begin{array}{l}\text { Inmaculada } \\
\text { Gil / Enrique } \\
\text { Herrera y Sara } \\
\text { Esteller }\end{array}$ \\
\hline $\begin{array}{l}\text { El círculo } \\
\text { de la danza. } \\
\text { Revista } \\
\text { de Artes, } \\
\text { Ciencia y } \\
\text { Movimiento }\end{array}$ & 2005 & 2005 & 3 & $\begin{array}{l}\text { ISDAA- } \\
\text { Universidad } \\
\text { Rey Juan } \\
\text { Carlos }\end{array}$ & $\begin{array}{l}\text { Alberto García } \\
\text { Castaño }\end{array}$ \\
\hline Danzaratte & $2 / 2006$ & vigente & 8 & $\begin{array}{l}\text { Conser- } \\
\text { vatorio } \\
\text { Superior de } \\
\text { Danza de } \\
\text { Málaga }\end{array}$ & $\begin{array}{l}\text { Dolores } \\
\text { Vera, Miguel } \\
\text { Carrasco, Ana } \\
\text { Alises }\end{array}$ \\
\hline
\end{tabular}




\begin{tabular}{|l|l|l|l|l|l|}
\hline $\begin{array}{l}\text { En } \\
\text { Moviment. } \\
\begin{array}{l}\text { Reflexiones } \\
\text { en torno a la } \\
\text { danza }\end{array}\end{array}$ & $10 / 2006$ & & 5 & $\begin{array}{l}\text { Mercat de } \\
\text { les Flors }\end{array}$ & $\begin{array}{l}\text { Joaquín } \\
\text { Noguero/ } \\
\text { Bárbara } \\
\text { Raubert }\end{array}$ \\
\hline
\end{tabular}

Tabla 1. Publicaciones españolas especializadas en danza (1972-2015)

[Elaboración de la autora, a partir de las fuentes reseñadas]

\section{Referencias}

Aviñoa Pérez, Xosé. 1987. "Publicistas". P. 220 en Historia de la Danza en Cataluña, textos: Pilar Llorens et al. Barcelona: Caixa de Barcelona

Brozas Polo, María Paz, Teresa García San Emeterio y Sara López Azcuna. 2011. "La danza contemporánea en España (1989-2009): Aproximación a la creación coreográfica a través de la revista 'Por la Danza'". Retos: Nuevas Tendencias en Educación Física, Deporte y Recreación 20: 16-20. http://www.retos.org/numero 20/RETOS\%2020\%2016-20.pdf

Bravo, Julio. 1992. "Variaciones" $A B C, 27$ de septiembre

Elvira Esteban, Ana Isabel. 2012 “La investigación en danza ¿Hacia dónde vamos?”. Por la Danza 95: 86-89

García Otzet, Montse. 1993. "Dansa 79, entusiasta ball de lletres". Assaig de Teatre 66-67: 118-122

Salas, Roger. 2013. "¿Cómo se escribe la historia de la danza?” El País, 11 de enero

- 1987. "Desaparece la única revista española de danza y 'ballet'” El País, 11 de enero

- 1986. "Carme del Val y Montse G. Otzet." El País, 22 de julio

Vivancos, Gala. 2009. "Pilar Llorens, 'Pastora Martos'”. Dectatoma: Revista Musical/Biografías. Ultima actualización 14 de diciembre. http://babourc.eu/Resources/dectatoma/todoarte pastora martos.htm

Notas

${ }^{1}$ Para la realización de esta investigación se han consultado los fondos del Centro de Documentación de Música y Danza, el archivo de la Asociación de Profesionales de la Danza en la Comunidad de Madrid y la Asociación Cultural Por la Danza y mi archivo personal. Quiero agradecer la generosa ayuda ofrecida por Rosana López en el CDMyD y William Arroyo en la ACPD. Revistas consultadas: ADE; ¡Bailar!; Ballet 2000; Cairón; Con D de Danza; El Círculo de la Danza; Dansa 79; Dansart; Dantzariak; La Danza; Danza Ballet, revista de colección; Danza Europa; Danza en Escena; Diálogos de Danza; E-danza. net; En Moviment; Inquedanzas; Melómano; Monsalvat Danza; El Pateo; Por la Danza; E Público; La Tarasca, Tiempo de Danza; Revista de Folklore; Ritmo; Scherzo; SusyQ y Zaradanza.

${ }^{2}$ Hasta ese momento, la publicación se presenta en su portada como "boletín informativo" y es a partir de esa fecha, que corresponde al $n^{\circ} 17$, cuando se denomina "revista especializada". 
${ }^{3}$ Nos referimos a los boletines de la Associació de Professionals de la Dansa de Catalunya (Bulleti y Kos), de la Associació de Professionals de la Dansa de la Comunitat Valenciana (Boletín informativo) y de la Asociación de bailarines, profesores y coreógrafos del País Vasco (Soka), entre otros.

${ }^{4}$ Esta revista se puede consultar en http://www.edanza.net

${ }^{5}$ El número 1 de Melómano Danza, se publicó en mayo de 1998, como suplemento gratuito.

${ }^{6}$ ARCA es el Archivo de Revistas Catalanas Antiguas, un portal de acceso abierto que incluye publicaciones periódicas cerradas que han sido representativas de la cultura y la sociedad catalana. Es un depósito cooperativo de preservación digital impulsado por la Biblioteca de Cataluña con el apoyo del Consorcio de Bibliotecas Universitarias de Cataluña (CBUC). Los ejemplares de Dansa 79 se pueden consultar y descargar en formato pdf http://www. bnc.cat/digital/arca/index.php?fname=titols/dansa79.html

(Artículo recibido 11-05-15; aceptado 09-06-15) 